(C)2007 IEEE. Personal use of this material is permitted. However, permission to reprint/republish this material for advertising or promotional purposes or for creating new collective works for resale or redistribution to servers or lists, or to reuse any copyrighted component of this work in other works must be obtained from the IEEE 


\title{
Interactive Online Learning Support: Progress on Research to Date
}

\author{
Dr Stephen QUINTON \\ Knowledge Networks and eLearning \\ Digital Ecosystems and Business Intelligence Institute (DEBII) \\ Curtin University of Technology \\ Perth, Western Australia \\ Email: s.quinton@curtin.edu.au
}

\begin{abstract}
This paper reports on progress to date in relation to a long term research project underway at Curtin University of Technology. The project aims to devise tools that interactively support the cognitive learning processes of today's knowledge workers as exemplified by researchers, managers and students. The empowering tools alluded to here are not simply intended to manage, calculate, search, order and arrange search results as is often the case with current search systems. Instead, the project is concerned with applying semantic analysis techniques, specifically the Normalised Word Vector (NWV) technology developed at Curtin to the construction of a range of 'intelligent' online cognitive support tools. The goal is to devise a system that enhances learners' cognitive functioning by augmenting the human intellect to achieve greater capacity to conceptualise and ultimately, construct new knowledge.
\end{abstract}

Index Terms - online learning, cognition support, interactive feedback

\section{Introduction}

Whenever a search is conducted on the web (or computer network), it is often the case that the displayed results represent little more than a collection of unrelated, unstructured information that has been extracted from its original context. The problem that confronts the learner is to organise the results and then apply the appropriate processing strategies to identify any meaningful connections that may assist to derive potentially useful solutions to a known problem or learning need. What is required is an 'intelligent' cognitive support system that invites input from the learner (a query for example), then locates, assembles and presents a collection of information based on the concepts contained within the query. The system then elicits further input and presents feedback (both visual and textual) in an interactive process that is iteratively analysed and refined until the learner's input indicates that a meaningful learning outcome has been attained (an interrogative process).

It is conceivable that web-based learning environments will one day be supported by software agents that are programmed to assist humans form new associations between concepts, synthesise information in the creation of new knowledge, and to solve problems on demand. This type of cognitive 'immersion' in online information and content repositories is at present within the realms of possibility. This paper outlines the rationale and theory behind a research project that aims to evolve web-based teaching environments into 'thinking' systems that respond 'intelligently' to human input. Rather than being a convenient tool for accessing information, it is argued that to be truly effective, online learning solutions must interact with students to support their conceptual thinking and cognitive development processes. The objective is to provide 'intelligent' support tools that will assist online learners to achieve deep learning.

The research project is premised on the notion that for learning to be truly effective, something much more complex than competence in information management skills is vital for ensuring learners achieve deep understanding. That is, the online learning environment should not only equip learners to quickly locate and sort through vast quantities of data and information, but also support them to apply complex analysis techniques and higher order thinking strategies to the construction of knowledge. Although there are tools that indirectly assist to enhance conceptual understanding such as concept maps, topic maps, and the Compendium project, their usefulness is limited in that they are incapable of providing the complex levels of interactivity that guide learners to identify key relationships and ultimately derive new meanings. In other words, they do not interact directly with learners to augment and empower the human intellect. In learning theory terms, the research project aims to facilitate a constructivist learning approach as students are encouraged to access appropriate information, refine it to suit the intended learning goal, structure it to match their individual cognitive frameworks, learning styles and preferences, test goal attainment and progression through evaluation and reflection, and then move on to the next goal.

\section{The Educational Efficacy of Cognitive Support Tools}

In today's web-focussed world, experienced researchers understand that the problem with the available search tools is to know exactly what to enter into the keyword criteria field. If the categorised terms or keywords are provided as a dropdown list then exploration of the search results can be confined to a manageable information set. This strategy is highly suited to ensuring students are exposed to information that support attainment of predetermined learning outcomes. If however, the task is to discover or generate something new or innovative ideas, that is to create knowledge, then the use of restrictive search strategies is highly unproductive. For example, a search on most knowledge repositories will yield hits related to the established terms, constructs and concepts unique to specific domains, and therefore prove unhelpful for the 
individual working in another discipline who seeks understanding on the interconnections that are emerging across different domains. In many instances, the researcher is confronted with the time consuming task of 'processing' large quantities of what may or may not be potentially relevant items. It is in this regard that current information retrieval systems provide minimal assistance where it is expected that research and learning should progress in the light of new and expanded conceptual understandings.

If we look to the recent past for clues as to how the problem of information overload has been dealt with, educational theorists and cognitive psychologists Ausubel [1] and Ausubel, Novak, \& Hanesian [2] advocated the use of "advance organisers" to foster meaningful learning and assist in managing information. Advance organisers are global overviews of the material to be learned that prompt the learner to recognise the pre-existing superordinate concepts formed within their own cognitive schema. This approach emphasises a tangible context for identifying the most general concepts into which the student can incorporate progressively differentiated facts and concepts. In theory, advance organisers are most effective if they make explicit the relationships amongst the concepts that learners already know and provide a structure or context in which to integrate new concepts.

Since Ausubel's time, many knowledge (or memory) management tools and visualisation (or browsing) software tools have been developed, most of which can be grouped into several generalised categories: Concept Mapping tools [6], [23]; Semantic Networking tools [12]; Cognitive Maps [11]; Topic Maps [24], [22]; Mind Maps [5]; Compendium [8]; 'intelligent' personal assistants [3]; and, decision making tools (also called group organisers). Canas et al. [6] noted a considerable overlap among these categories as most tools support the visual representation of knowledge as expressed in terms of concepts, ideas or "thoughts"; the associative creation of links and nodes; and, the capacity to organise related concepts for specific purposes. A brief description of the most common examples of cognitive support tools is provided as follows:

- Concept Maps are useful for organising and representing knowledge by assisting the user to consciously and explicitly link known concepts to new knowledge in a diagrammatic form. Canas et al [6] hold the view that evidence of usefulness, usability, performance enhancement, or organisational effectiveness has not yet been conclusively provided.

- The Semantic Web project (W3C http://www.w3.org/) was established to devise new ways to define and link information on the Web for automation, integration and reuse across applications. The project aims to create a universal medium for information exchange by giving meaning (semantics) to the content of web-based documents.

- A topic map is a document that conforms to a model which is designed to improve information retrieval and navigation using topics as hubs in an information network. Topic maps are used to assist people find information quickly and easily. They can be formatted into a wide variety of search aids such as printed indexes and glossaries [22].
- The Compendium project [8] comprises three key elements: a shared visual space where ideas can be generated and analysed, a methodology that permits exploration of different perspectives, and a set of tools for quickly and easily sharing data both within and beyond the boundaries of a group. Compendium's mapping technique for group discussions, combined with its interoperability with domain-specific applications, provides a useful platform for knowledge construction and negotiation.

- The Barr et al. [3] review of Smart Internet Technology appraises a number of applications for software agents amongst which they note the 'personal assistant' is already being realised through applications that aid in information processing (such as personalised web searches and information aggregation), communication processing (email, document management, and call handling), and time management (scheduling applications).

Over the past several decades, the focus of artificial intelligence (AI) research has been on how computer technology can be harnessed to achieve machine intelligence. Minsky [21] advanced the view that knowledge is an artefact that can be stored in a machine and it is the application of that stored knowledge to the real world that constitutes intelligence. This 'realist' viewpoint underpins much of the research in AI that has been undertaken to date and has given rise to the notions of semantic networks and rule-based (AI) expert systems. However, Maes [19] makes a valuable distinction between technology-reliant artificial intelligence and intelligence augmentation (IA) that aims to enhance the learning process. For Maes, software agents of the future will conduct negotiations on behalf of users and prevent information overload through the identification of new patterns within information. The distinction made by Maes points to a crucial factor when considering the design of technology supported learning systems.

Jonassen \& Wang [16] concluded that merely providing content and information, and revealing structural relationships to learners is not sufficient for improving higher order cognitive performance. They point out that "what matters most is the construction of personally relevant knowledge structures" (p 7). This means that learners must be capable of accessing resources that match or accommodate their individual preferences and goals, whilst being encouraged to engage with the displayed learning materials at varying levels of complexity. Although in principle this approach is conducive to establishing effective learning experiences, when it comes to the use of computer technology, the research literature reveals that most existing systems and tools are characterised by a number of limitations:

- when undertaking a specific enquiry for information, often it is necessary to search the entire Web and manually abstract data that may hold some conceptual relevance

- vast quantities of research information held in different databases around the world are not structured. No generative infrastructure is available to 
assist in managing, searching, translating, categorising, indexing, and accessing the exact information required.

- $\quad$ at present, most search results return unmanageably large lists of potentially relevant documents. An opportunity exists to provide 'generative support' that facilitates overarching and exhaustive analyses of multiple relevant factors.

- to be effective, the researcher must possess an unreasonably large amount of domain related knowledge. Knowledge pertaining to who, what, where, and current developments can be readily dealt with by technology and yet this task is still being carried out by the researcher.

- whereas portions of the information or data on the Internet may be related to each other, other information may overlap or is semi-complementary. At present, no knowledge-based middleware is available to manage semantic connections.

Jonassen \& Reeves [15] confirmed it is possible to design cognitive tools that assist learners to organise, restructure and represent what they know and that such tools can support their investigations to enhance the learning process. Other researchers favour extending or changing learners' cognitive systems and their preferred learning approaches through the use of adaptive, intelligent computer courseware and learning materials [14]. Wobcke's 'Intelligent Agents Technology Review' [30] for example, canvasses a number of applications for software agents in which he notes that ultimately, the goal of implementing the notion of a 'smart system' is demonstrated through its capacity to reason and think about itself and its environment. Where learning is concerned, such a system must focus on the needs of students and in some respects take on the role of facilitating cognitive support similar to that of a personal tutor. Wobcke [30] further observed that "more complex personal assistants aim to address more than one of these aspects within a single system". An intelligent system of the type described here introduces a core component of the research project in that it needs to be 'metacognitively aware' of all its components, be capable of interacting with the learner by analysing the myriad of needs that arise, whilst learning and adapting as the learner grows, learns and adapts. Thus, learning environments in the future should incorporate principles of self-organisation and networked systems theory as applied to understanding how to design and manage multiple levels of complexity that continually (and automatically) interact to deliver more comprehensive, deeper learning experiences.

\section{The Research Project}

The goals of the Interactive Online Learning Support project are premised on the notion that in order to facilitate effective online learning environments, the functionally of current cognitive support tools must be extended to include the enhancement of metacognitive thinking skills through the provision of direct 'intelligent' interaction with individual learners. It is further argued that if online learners are to become skilled in deriving new meanings and understandings, software designers need to provide systems that interact with the investigative process and provide immediate feedback on all activities. A parallel requirement of the project is that such tools must be readily incorporated into existing learning delivery systems and interoperate with any electronic content and natural language input. To this end, the project builds on the Normalised Word Vector (NWV) technology devised by Williams [26] to determine how learners can be assisted to gain deep cognitive insights whist engaged in electronic learning environments. The aim is to empower learners to perform tasks more accurately and intelligently by interactively applying their personal knowledge and experiences to improving their information search and independent learning capabilities. The outcomes will serve as 'proof of concept' and thereby provide the basis for deriving a new theoretical foundation on which future online design strategies can be devised.

The primary challenge to be addressed in this project will be to derive a technology solution that on receipt of human input generates the most appropriate categories, highlights areas of relevance by displaying visual representations of identified associations, dynamically forms links to new materials, stores derived links and access trails, and juxtaposes what previously had not been associated with new content. All the while, the ephemeral (often tedious) task of recalling past actions is managed by the system's recording and storage capabilities, which also scan the learner's responses to the given information and the links that were made during the process. Such a system supports what the human mind does naturally by dynamically assisting the learner to make sense of information. The high level of complexity and interaction indicated here further emphasises the need to devise 'intelligent' cognitive support tools.

A learning support system will be devised that acts to 'interpret' inert, decontextualised information and present the results to the learner in an iterative process that compares their responses with a preferred model answer and displays visual feedback by way of graphs, concept maps, and statistical data. This process continues until a match occurs that is within a predefined level of accuracy. For example, a bar graph that compares the 'concepts' associated with the model answer and the student's answer, would in the case of a close match, indicate that the student has understood the required concept. Where the student's response does not match the model answer, either the student has made an error, has not learned the concept, or introduced irrelevant material. Visual feedback of this type is immediate and highly informative to both student and lecturer [13]. What have been described to this point are the key principles underpinning what Dreher [9] refers to as the Generative Conceptualisation and Cognition Support System (GCCS) model.

There are four aspects of Dreher's GCCS model to be applied to this project. At the outset of a learning activity, the first task is to identify the 'information space' from which learners are expected to derive new knowledge and represents the unordered collection of information (for example, journal articles) that is to be 'generatively 
processed'. This information is scanned by the NWV analysis engine to identify a broad categorisation of concepts to be targeted for further exploration. Next, a graphical representation of the targeted and related concepts acts as a visual stimulus that forms a feedback bridge in that it provides an elaboration/explication of the concepts found in amongst the scanned information. Third, the cognitive dissonance aspect of the Generative Conceptualisation model is achieved by 'highlighting' the identified concepts that align most with the concepts to be learned and associatively linking them with related concepts that serve as prompts for encouraging further understanding and learning. The identification of the concepts to be acquired by the learner is accomplished using NWV analysis of a preferred model answer. Finally, cognitive activity progresses in a recursive manner until the learner generates a response satisfactory to the intended learning goals.

In this approach, concepts are the embodiment of the knowledge being created or synthesised by the user. Once identified, they are superimposed onto and dynamically linked to a set of related articles and documents. In essence, a concept is viewed as 'knowledge about knowledge' or meta-knowledge, which is in contrast to the focus of most cognitive support or mapping tools that serve to identify existing concepts as opposed to the highlighting of associative links that further assist in the creation of new concepts. Thus, in this application a model of interactive enhancement of learners' metacognitive thinking skills is described where the metacognitive process is defined as thinking about thinking which embraces the need for learners to know what they know as well as what is not known [4].

The strength of the NWV technology resides in its ability to 'normalise' all words contained within an electronic document. An electronic thesaurus is employed to build vector representations of the concepts that are inherent within each word found in a document (or information space). That is, vector algebra techniques are employed to mathematically represent conceptual similarities within the given content. The words are normalised by comparing and reducing each to the most appropriate thesaurus root word. It is this root word (or concept) that is used as the basis for comparison with the model answer.

The normalised concepts are derived from the Macquarie Thesaurus [18]. Any electronic thesaurus is suitable for use by this technology, thus extending functionality and application. There are 812 concepts in the Macquarie thesaurus and all words in the document are reduced to the most closely aligned 'within context' root concept. The vectors are constructed in this 812 dimensional space where the principles of vector theory apply in exactly the same way as the $\mathrm{x}, \mathrm{y}, \mathrm{z}$ dimensions familiar to most people. The vector representations are constructed by counting the number of times a word in the document matches the thesaurus root concepts. Each dimension consists of a concept number which is then compared to the vector representations calculated for the model answer. The angle between each vector indicates the level of accuracy. If the angle is small, the document contains content similar to the model answer. Conversely, the greater the angle the less accurate the match between the corresponding vectors [26] (pp 3 -4). The advantage of using NWV as opposed to latent semantic analysis (for example), is that it is computationally more efficient and therefore requires less time to process. Complete descriptions of various NWV technology applications are described in Williams \& Dreher [27], Guetl, Dreher, \& Williams [13], Williams \& Dreher [28], and Dreher [10]

The task of assembling new teaching content will be accomplished using two interdependent techniques. The first applies to the dynamic selection of resources (learning objects) from existing content repositories based on criteria that are recorded and stored as the learner progresses through the learning environment. It is envisaged that the search criteria (or combination thereof) will be drawn from a number of sources: the automatic construction of learner profiles in relation to users' preferred cognitive styles, learning preferences, and changing performance levels; the required learning outcomes using assessment rubrics; user input, interaction and associated learning needs analysis; and, the full spectrum of current learning theories as applied to delivery sequencing, presentation format and the level of learner independence that is permitted for each designated learning activity.

The second method applies to the automatic generation of learning objects from non-learning object compliant electronic materials (documents, reports, papers), thus removing the need for prior conversion to an XML compliant format. Dynamic metatags are derived and generated 'on-the-fly' in response to the search criteria determined by the NWV engine to be the most accurate 'conceptual' match for the selection of suitable learning object material. Thus, dynamic metatag generation, dynamic object selection, and NWV technology will function as mutually dependent processes.

In arguing that the field of computational intelligence can contribute to web-based technologies, methods and techniques that directly support online learning, Karampiperis and Sampson [17] propose two methods for automatic content (learning object) selection and sequencing: Adaptive Courseware Generation where the entire lesson or activity is adaptively generated according to the required learning outcomes and learners' knowledge levels which are defined before presentation; and, Dynamic Courseware Generation in which the system monitors the 'student's progress and dynamically modifies the displayed content in response to students' learning needs taking into account their individual cognitive styles, experiences, previous performances, and preferences. However, as Karampiperis and Sampson acknowledge, whilst technically these approaches hold real promise for adaptive navigation and courseware delivery, they are not completely ideal when it comes to the practical realities of producing effective online teaching solutions. In their view, there are no well-defined, universally accepted rules on how learning objects should be selected and sequenced in order to inspire educationally sound learning experiences. The problem as they see it, is that the design of highly adaptive learning systems requires an enormous set of rules to cope with the complex dependencies between the 
educational attributes of learning objects and the learning needs of students.

With the preceding issues, needs and potential directions in mind, the developmental approach to be observed as this project progresses will be to investigate the feasibility of combining the principles of automatic content selection and sequencing as described by Karampiperis and Sampson, with the techniques of dynamic learning object selection and dynamic metatag outlined beforehand, to determine how the key attributes of each approach can be applied in response to the decisions that are made by the Normalised Word Vector engine. A core component of this research study therefore, will be to devise a design model for the automatic generation of metatag sets based on the concepts identified by the NWV engine that in turn trigger the selection of learning object content in a process that is optimised to the learner's needs each time they undertake an inquiry or respond to a learning activity. The concepts identified within the scope of an inquiry or activity are interpreted by the NWV engine which seeks out, temporarily tags, and hands control over to a content management system (CMS) that assembles selected learning object content to inform/teach/report in accordance with the learners' needs. In this way, it is possible to locate, modify, and/or expand on the displayed teaching content as the learner forms new ideas and understandings.

To summarise to this point, the research study has employed an interdisciplinary approach to applying dynamic learning object generation that is combined with 'concept orientated' dynamic meta-tags that are generated in response to user input. Key concepts interpreted by the NWV technology provide the 'normalised concepts' that are used to assemble information and content in direct response to learners' immediate needs. Where online learning is concerned, the time-consuming task of creating or locating suitable teaching material will be significantly reduced.

\section{Future Directions}

Information and Communications Technologies (ICT) have pervaded our society to the degree that a growing proportion of human activity is now knowledge work in contrast to physical work. The history of empowerment of physical workers through technology clearly demonstrates the enormous benefits to be gained in terms of increased production and the efficient utilisation of resources. Learners should also expect similar gains in thinking capacity, productivity, and in the quality of their learning outcomes. As an example, software support systems could be designed to allow users to make more decisions about how their learning goals are met by guiding their interactions with 'intelligently' refined teaching resources. There are several ways this could be achieved. In one scenario, learners could make decisions (with varying degrees of guidance) about both content (what to learn) and strategy (how to learn it). They can then apply adaptive, computer-managed, 'intelligent' learning tools to refine their own knowledge and understanding of a subject area. Learners could also request the system to modify the content display by selecting from a range of computer initiated options that are derived through continual analysis of their input. For example, learning object resources could be dynamically assembled to generate customised responses based on progress, areas of difficulty, or the need for revision. To illustrate further, a number of strategies can be applied: alert the student to the need for revision and present appropriate alternative material; require the student to repeat a set learning sequence using new materials; dynamically generate quizzes, assignments, or exercises to determine the student's comprehension levels and immediate learning needs; and evaluate students' comprehension levels to provide just-in-time feedback [25]. In the words of Winn [29]:

..It follows that the only viable way to make decisions about instructional strategies that meshes with cognitive theory is to do so during instruction using a system that is in constant dialogue with the student and is capable of continuously updating information about the student's progress, attitude, expectations, and so on.

It is the view of all project team members that this research project will revolutionise the use of information repositories and lead to marked improvements in the quality and depth of learners' knowledge. The benefits to be derived from a cognitive support system as described in this paper can be applied to many fields of endeavour. The project focuses on the application of ICT to enhance human cognitive processing as an aide in knowledge innovation, and to advance learning and thinking skills. As such, the outcomes are expected to advantage all members of society from the individual through to business organisations and government administrations. For learners, the main benefits will include the capacity to learn on demand and derive solutions to problems in a way that best suits their current understandings and experiences; for governments and organisations, the provision of 'intelligent' support technologies will lead to increased production and innovation; a measurable capacity to apply existing knowledge in imaginative ways; calculable cost advantages and procedural efficiencies; and finally, enhanced contributions to achieving organisational goals.

In general terms, the benefits to be realised apply to: an increased capacity for individuals and organisations to acquire and generate new knowledge; social gains in terms of increased opportunities to participate in collaborative knowledge building activities; and a more effective information management capacity in a world characterised by exponential increases in readily accessible information. A significant aspect of this project is that it presents a unique opportunity to identify and establish the ground rules from which the benefits of applying technologies to enhance conceptual thinking can be realised. Successful completion, coupled with other related research activities currently underway at Curtin, will result in a robust development methodology capable of producing cognitive support systems for applications that currently have not been made available due to technological limitations. For the present, the main contribution to knowledge is to increase current understanding on how learning may or may 
not be supported and enhanced using technology. The final words go to Martinez [20] whose perceptive analysis of the future of online learning is conclusively encapsulated in the following extract:

The Web offers the perfect technology and environment for individualized learning because learners can be uniquely identified, content can be specifically personalized, and learner progress can be monitored, supported, and assessed. Technologically and technically, researchers are making progress toward realizing the personalized learning dream with adaptive learning object technology.

\section{Reference List}

[1] Ausubel, D. P., Educational Psychology: A Cognitive View, New York: Holt, Rinehart and Winston, 1968.

[2] Ausubel, D. P., Novak, J. D. and Hanesian, H., Educational Psychology: A Cognitive View (2nd ed.), New York: Holt, Rinehart and Winston, 1978.

[3] Barr, Trevor., Burns, Alex., and Sharp, Darren., Smart Internet 2010 , Faculty of Life and Social Sciences, Swinburne University of Technology. Report produced for the Smart Internet Technology CRC Pty Ltd, Australian Technology Park, Eveleigh, NSW, Australia., 2005. WWW Ref.

http://www.smartinternet.com.au/SITWEB/publication/publications.j $\underline{\mathrm{sp}}$

[4] Blakey, Elaine and Spence, Sheila, Thinking for the Future., Emergency Librarian, 17:5, May-June, 1990, 11-14.

[5] Buzan, T. and Buzan, B., The Mind Map Book: How to Use Radiant Thinking to Maximize Your Brain's Untapped Potential, Plume, 1996.

[6] Canas, Albert., Coffey, John. W., Carnot, Mary Jo., Feltovich, Paul., Hoffman, Robert R., Feltovich, Joan., and Novak, Joseph D., A Summary of Literature Pertaining to the Use of Concept Mapping Techniques and Technologies for Education and Performance Support, Report prepared by The Institute for Human and Machine Cognition and The University of West Florida for The Chief of Naval Education and Training, Pensacola FL., 2003, 60. WWW Ref. http://cmap.ihmc.us/Publications/

[8] Compendium [Online], January, 2006. WWW Ref. http://www.compendiuminstitute.org/

[9] Dreher, H., Empowering Human Cognitive Activity through Hypertext Technology, PhD thesis, 1997. WWW Ref. http://adt.curtin.edu.au/theses/available/adt-WCU2000046.121219/

[10] Dreher, H., Interactive On-line Formative Evaluation of Student Assignments, Presented at InSITE 2006, Greater Manchester, England, June, 2006, 25-28, WWW Ref http://2006.informingscience.org/

[11] Eden, C., Cognitive Mapping, European Journal of Operational Research, 36, 1988, 1-13.

[12] Fisher, K.M., SemNet Software as an assessment tool, Assessing Science Understanding, 2000, 197-221.

[13] Guetl, C., Dreher, H. and Williams, R., E-TESTER: a Computerbased Tool for Auto-generated Question and Answer Assessment, In Proceedings of World Conference on eLearning in Corporate, Government, Healthcare, and Higher Education, E-Learn, October, 2005, 2929-2936. WWW Ref: http://www.editlib.org/index.cfm
[14] Jonassen, D., Integrating learning strategies into courseware to facilitate deeper processing, In D. Jonassen (Ed.), Instructional designs for microcomputer courseware, Hillsdale, NJ, Lawrence Erlbaum Associates, 1988, 151-181.

[15] Jonassen, D. H., and Reeves, T. C., Learning with Technology: Using Computers as Cognitive Tools, In D. H. Jonassen, (Ed.) Handbook of Research on Educational Communications and Technology, New York Simon \& Shuster Macmillan, 1996, 693-719.

[16] Jonassen, D. H., and Wang, S., Acquiring structural knowledge from semantically structured hypertext, Journal of Computer-based Instruction, 20:1, 1993, 1-8.

[17] Karampiperis, Pythagoras and Sampson, Demetrios., Adaptive Learning Selection in Intelligent Learning Systems, Journal of Interactive Learning Research, 15:4, 2004, 389 - 407.

[18] Macquarie Library, 2006, WWW Ref. http:/Www.macquariedictionary.com.au

[19] Holloway, Marguerite., Pattie, Wired Magazine, December, 1997. WWW Ref: $\underline{\text { http://Www.wired.com/wired/archive/5.12/maes.html }}$

[20] Martinez, M., Designing learning objects to personalize learning, In D. Wiley (Ed.), The Instructional Use of Learning Objects, Indiana, Agency for Instructional Technology and Association for Educational Communications \& Technology, 2001.

[21] Minsky, Marvin., (Ed)., Semantic Information Processing., Cambridge, Massachusetts, The MIT Press, 1968.

[22] Newcomb, Steven R. and Biezunski, Michel., Topic Maps go XML, 2000. WWW Ref. http://www.gca.org/papers/xmleurope2000/pdf/s11-02.pdf

[23] Novak, J., The Theory Underlying Concept Maps and how to Construct Them, circa 1999. WWW Ref. http://cmap.coginst.uwf.edu/info/

[24] Pepper, S., The TAO of Topic Maps: Finding the Way in the Age of Infoglut, 2000. WWW Ref. http://www.ontopia.net/topicmaps/materials/tao.html

[25] Quinton, S., Towards Dynamically Generated, Individualised Learning, Refereed paper accepted for publication in e-University: International Conference on ICT and Higher Education, Dr. Wichian Premchaiswadi (Ed.). Siam University, Bangkok: Thailand, 2004.

[26] Williams, R., The Power of Normalised Word Vectors for Automatically Grading Essays, Presented at InSITE, Greater Manchester, England, June 25-28, 2006. WWW Ref. http://2006.informingscience.org/

[27] Williams, R. and Dreher, H., Automatically Grading Essays with MarkitC, Issues in Informing Science and Information Technology, (1), 2004, 693-700. WWW Ref. http://articles.iisit.org/092willi.pdf

[28] Williams, R. and Dreher, H., Formative Assessment Visual Feedback in Computer Graded Essays. The Journal of Issues in Informing Science and Information Technology, 2, 2005, 23-32. WWW Ref. http://2005papers.iisit.org/103f95Will.pdf

[29] Winn, W., Toward a rational and theoretical basis for educational technology. Educational Technology Research \& Development, 37:1, $1989,35-46$

[30] Wobcke, Wayne., Intelligent Agents Technology Review. Smart Internet Technology CRC, Sydney, October, 2004. 